Annuaire suisse de politique de développement

26-2 | 2007

Financer le développement par la mobilisation des ressources locales

\title{
Les fonds de garantie, une pratique de solidarité et de mobilisation des ressources pour le développement
}

Jean-Michel Servet

\section{CpenEdition}

\section{Journals}

Édition électronique

URL : http://journals.openedition.org/aspd/138

DOI : 10.4000/aspd. 138

ISSN : 1663-9669

Éditeur

Institut de hautes études internationales et du développement

Édition imprimée

Date de publication : 1 novembre 2007

Pagination : 143-156

ISBN : 978-2-88247-068-3

ISSN : 1660-5934

\section{Référence électronique}

Jean-Michel Servet, «Les fonds de garantie, une pratique de solidarité et de mobilisation des

ressources pour le développement », Annuaire suisse de politique de développement [En ligne], 26-2 I

2007, mis en ligne le 22 juin 2009, consulté le 07 septembre 2020. URL : http://

journals.openedition.org/aspd/138; DOI : https://doi.org/10.4000/aspd.138 


\title{
Les fonds de garantie, une pratique de solidarité et de mobilisation des ressources pour le développement
}

\author{
Jean-Michel Servet*
}

es ressources courantes des institutions pratiquant le microcrédit ${ }^{1}$ peuvent provenir de l'épargne des emprunteurs dans un système à caractère mutuel ou sous la forme d'une caisse d'épargne-crédit, des ressources propres d'un établissement financier, de dons publics de coopérations multilatérales ou bilatérales ou de fonds privés provenant d'organisations non gouvernementales, de fondations ou de crédits mis à la disposition des institutions de microfinance et de prises de participation ou de prêts par des fonds d'investissement. Parmi leurs ressources exceptionnelles, on doit évoquer aussi le cas des fonds d'urgence, qui peuvent être mobilisés par une organisation de microfinance en cas de catastrophes naturelles soudaines telles qu'ouragans, inondations, tremblements de terre, etc., qui détruisent une large fraction des moyens de travail et de vie des clients ou membres des organisations de microfinance et des organisations de microfinance elles-mêmes ${ }^{2}$. Il s'agit là de crédits consentis pour faire face à des pertes dans des circonstances exceptionnelles ${ }^{3}$ et non d'un fonds de garantie à proprement parler. Toutefois, ces prêts agissent comme une garantie pour l'organisation de microfinance et, selon certains acteurs, ils diminuent la vigilance des organisations de microfinance pour intégrer dans l'évaluation des risques la probabilité des catastrophes. On peut considérer que ces fonds d'urgence sont nécessaires pour assurer une pérennité aux institutions de microfinance intervenant dans des zones aux conditions climatiques ou politiques difficiles. Il convient ici de rappeler le rôle essentiel que la microfinance est capable de jouer dans les situations postcrise ou postconflit ${ }^{4}$.

* Professeur à l'Institut universitaire d'études du développement (IUED), Genève.

1 Cette contribution à l'Annuaire développe certains arguments présentés dans Jean-Michel Servet, Banquiers aux pieds nus. La microfinance, Paris, Odile Jacob, 2006, pp. 252-256, ainsi que les communications faites dans le cadre du Réseau AUF Entrepreneuriat à Rouen en avril 2006 et à Antananarivo en mai 2007 et dans le cadre des $4^{\text {es }}$ Rencontres interuniversitaires de l'économie sociale et solidaire en juin 2006 à Grenoble. Je remercie les participants à ces colloques ainsi que Daniel Fino et Cédric Lombard pour les suggestions qu'ils m'ont faites après une lecture d'une première version de ce texte. Nous n'avons pas pu toutes les intégrer ici.

2 Les exemples les plus connus sont ceux du Fundusz en Pologne après les inondations de 1997, un fonds mis en place en Amérique centrale par la Banque interaméricaine de développement (BID) après le cyclone Mitch, et les fonds de crédit d'urgence mobilisés après les inondations de 1998 au Bangladesh par le Bangladesh Rural Advancement Committee (BRAC), Buro Tangail, CARE et la Fondation Palli Karma-Sahayak (PKSF).

3 Le fonds de garantie d'Etika, fondé pour soutenir des opérations intégrant fortement une perspective de développement écologiquement soutenable, intervient par exemple aussi comme crédit-relais pour des institutions de microfinance en attente du versement de subsides publics.

4 Un des éléments les plus surprenants dans les attendus du Comité du prix Nobel attribuant le prix au professeur Yunus et à la Grameen Bank, qui illustre une méconnaissance répandue de la réalité 
Les fonds de placement, quant à eux, fournissent des ressources aux institutions de microfinance en s'appuyant soit sur une démarche éthique d'investissement ou de prêt solidaire, soit sur la recherche de placement à fort risque et à forte rentabilité ${ }^{5}$. Un des cas les plus souvent cités de ces fonds à risque est BlueOrchard à Genève ${ }^{6}$. Les placements éthiques qui s'inscrivent dans ce type de relation peuvent être considérés comme faisant partie de l'économie sociale et solidaire.

Le fait de privilégier l'approche crédit en microfinance est généralement fondée sur l'idée largement répandue que les populations pauvres manquent de ressources monétaires et que celles-ci doivent leur être procurées de façon externe. Ce qui est largement vrai dans les situations postcrise et postconflit ne l'est certainement pas de façon permanente parmi les populations aux revenus les plus faibles de la planète. De ce point de vue, le microcrédit rompt peu avec les logiques développementalistes de la seconde moitié du $\mathrm{Xx}^{\mathrm{e}}$ siècle.

Une autre logique est possible, fondée sur l'idée que les banques locales, bien que se trouvant souvent en situation de surliquidité ${ }^{7}$, refusent de courir le risque de microprêts et que le soutien extérieur doit porter moins sur l'apport de fonds que sur celui de garanties. Une garantie est un instrument financier protégeant le prêteur contre le risque de défaillance de l'emprunteur: si celui-ci ne rembourse pas, le prêteur peut ainsi être remboursé en totalité ou en partie (selon les termes de l'accord de garantie).

contemporaine de la microfinance en général et du microcrédit en particulier, est qu'aucune référence n'y est faite au rôle essentiel du microcrédit dans les situations postconflit. Or, cette technique financière originale a été un facteur de retissage des liens sociaux au Cambodge, en Ouganda, en Bosnie, au Rwanda..., mais aussi au Bangladesh. La Grameen Bank a été créée juste après la partition du Pakistan, qui avait plongé le nouvel Etat dans la guerre et une crise profonde. Le regard du Comité du prix Nobel est porté essentiellement sur les fondements économiques du microcrédit et de la pauvreté. Voir l'étude de Djimitri Edoé Agbodjan, La microfinance dans l'après-conflit, mémoire de DEA, Genève, IUED, 2006.

5 On peut citer divers exemples de fonds d'investissement actifs depuis plusieurs années : Profund, créé en 1995 à l'initiative de Calmeadow, d'ACCION International et de Fundes; la Société coopérative œcuménique de développement (SCOD), créée en 1975 aux Pays-Bas par le World Council of Churches; Dexia Micro Credit Fund, créé en 1998 au Luxembourg et géré par BlueOrchard; la Internationale Micro Investitionen Aktiengesellschaft (IMI), un fonds allemand; la Société internationale pour le développement et l'investissement (SIDI), créée en France par le Comité catholique contre la faim et pour le développement (CCFD), actionnaire de Profund en Amérique latine. Ils réalisent des prises de participation dans les institutions de microfinance. La SIDI est par exemple actionnaire de la Centenary Rural Development Bank (CERUDEB), en Ouganda.

6 Voir l'exemple d'un crédit de plus de 100 millions de dollars lancé par BlueOrchard en février 2006 pour prêter à 22 institutions de microcrédit actives en Asie du Sud, en Amérique latine et en Europe orientale, donné par Paul J. Davies, «Bond Gives Boost to Microfinance», Financial Times, 2 March 2006.

7 Selon l'étude suivante du Fonds monétaire international (FMI) : Magnus Saxegaard, Excess Liquidity and Effectiveness of Monetary Policy: Evidence from Sub-Saharan Africa, IMF Working Paper, WP/06/115, Washington, IMF, 2006, citée par Fatima Hmimid et Anselme Imbert, «Les banques centrales face aux défis du développement», Techniques financières et développement, $\mathrm{n}^{\circ}$ 83, juin 2006, p. 30. C'est par exemple le cas de 40 pays africains sur 44 où, du point de vue macroéconomique, l'épargne dépasse l'investissement. Pour absorber cette surliquidité bancaire, les banques centrales augmentent le taux de réserve obligatoire. On observe aussi des transferts vers le Nord où les rendements du capital placé sont estimés couvrir moins de risques et obtenir une rémunération supérieure. L'exemple africain de surliquidité bancaire le mieux documenté est celui du Maroc. Voir Jérôme Sgard, Rapport sur le risque-pays du Maroc, Document de travail, $\mathrm{n}^{\circ} 18$, Paris, Agence française de développement, juin 2006, 33 p. 
Un fonds de garantie, qu'il soit local, national ou international, est donc destiné à compenser les pertes éventuelles dans les opérations de crédit du fait de la défaillance des emprunteurs. Il existe plus d'une soixantaine de ces fonds agissant à une échelle internationale. Les fonds de garantie ont la particularité de mobiliser des ressources sans apport direct de fonds dans le prêt lui-même. C'est la raison pour laquelle ils ne figurent pas dans les statistiques de l'aide au développement ${ }^{8}$. Ces fonds constituent une modalité originale puisque la ressource de l'organisation de microfinance peut être obtenue à partir d'une confiance externe à l'institution financière accordant le prêt. Pour une institution à but lucratif, la diminution des pertes par ce mécanisme assurantiel, pris en charge par l'emprunteur, revient à accroître la rentabilité des prêts ou à en diminuer le coût (dans le cas de prêt subventionné). Les fonds de garantie peuvent aussi entrer dans une logique de développement de solidarités multipartites.

Après avoir précisé la définition des fonds de garantie, nous présenterons différentes modalités de leur fonctionnement ainsi que le cas du Fonds international de garantie (FIG) de Genève.

\section{Définition d'une garantie}

Une garantie est un instrument financier. Il protége un établissement faisant crédit en cas de défaillance de l'emprunteur. Un fonds de garantie est destiné à compenser les pertes éventuelles d'une institution faisant crédit dans l'octroi de ses prêts. Si l'emprunteur ne rembourse pas, le fonds s'étant porté garant doit assumer entièrement ou partiellement (selon ce que prévoit le contrat) les obligations de l'emprunteur. La garantie est donc la promesse d'un garant vis-à-vis d'un prêteur.

Les statuts des institutions apportant de telles garanties sont multiples. En Europe, on remarque que la garantie peut être apportée par les pouvoirs publics (par exemple en Belgique, aux Pays-Bas et au Royaume-Uni) ou par des associations professionnelles mutuelles (par exemple en Espagne, en France ou en Italie avec les confidi). Dans ce cas, la source de la garantie se trouve dans une mutualisation des risques entre emprunteurs puisque ce sont les bénéficiaires du crédit qui, sur une base professionnelle, se répartissent les risques de défaillance. Les établissements financiers prêteurs eux-mêmes peuvent organiser entre emprunteurs un fonds de garantie.

Ces modèles ne peuvent qu'exceptionnellement exister dans les pays en développement pour trois raisons essentielles. Les établissements bancaires y pratiquent généralement peu le crédit aux micro-entreprises et il est donc difficile d'imaginer un partage des risques organisé par un organisme bancaire. Le modèle mutualiste professionnel est difficilement transposable à court terme dans les pays en développement où n'existe généralement pas une tradition d'associations par métier' ${ }^{9}$ A noter que la concentration des prêts sur un même secteur d'activité tend à accroître le risque du crédit puisque les risques peu diversifiés sont eux-mêmes concentrés sur un même type d'activité; ils sont

Sauf pour la partie éventuellement utilisée en cas de défaillance de l'emprunteur.

9 Rappelons que dans de nombreux pays européens ce mutualisme et cet associativisme sont à l'origine des syndicats de salariés. 
donc moins compensés à l'échelle globale. Indépendamment d'un niveau de risque différent d'un secteur d'activité à l'autre, cette aversion pour des risques concentrés se traduit en microfinance par une préférence marquée des prêteurs pour des activités commerciales et de transport plutôt que pour des activités de production, et en particulier agricoles, supposant des investissements à moyen ou à long terme. La recherche d'une péréquation des risques se traduit aussi par la préférence pour prêter à une association de producteurs plutôt qu'à des producteurs individuels. Enfin, les garanties publiques sont souvent perverties par une gestion corrompue ou bureaucratisée, comme cela a été le cas dans la gestion des garanties de prêts aux agriculteurs dans de nombreux pays.

Dans le cadre des politiques de financement public ${ }^{10}$, des fonds de garantie, qui dépendaient généralement du budget des Etats, avaient été mis en place, principalement pour les prêts à l'agriculture. L'Inde illustre la faillite de tels fonds publics de garantie ${ }^{11}$. Dans les années 1980, deux fonds de garantie avaient été mis à la disposition des banques pour l'octroi de crédits aux petites entreprises; la participation à ces fonds était obligatoire pour toutes les grandes banques indiennes: pour tous les prêts inférieurs à l'équivalent de 580 euros, le système était gratuit et couvrait les neuf dixièmes du montant; la couverture a été ramenée ensuite à $60 \%$ du montant. Les frais s'élevaient à $0,5 \%$ du prêt. En fait, la plupart des banques remplissaient les formulaires d'appel de garantie dès l'octroi du crédit afin de les envoyer dans les meilleurs délais après le constat de la carence de l'emprunteur...

Au-delà de cet exemple de dysfonctionnement observé en Inde, il est aisé de comprendre les raisons de ces échecs passés. Des crédits, dont la probabilité d'être remboursés était faible, voire très faible, ont été octroyés parce qu'ils étaient couverts, quoi qu'il arrive, par la garantie. Le suivi des prêts était lacunaire lui aussi puisque en toute hypothèse ils étaient couverts. Cela se trouvait renforcé lorsque l'institution qui octroyait le prêt était la même que celle qui donnait la garantie (ou en étroite liaison avec elle); dans les années 1970-1980, cela a été largement le fait des banques de développement (octroyant des crédits agricoles) et des fonds de garantie sous contrôle étatique, dont la logique n'était pas d'établir des relations financières solides mais d'engendrer des effets multiplicateurs et de propager de nouvelles techniques de production et de nouvelles cultures. Ces échecs financiers ont répandu une attitude souvent extrêmement négative à l'égard des fonds de garantie. Jusqu'à une période récente ont donc été négligées les potentialités offertes par les fonds de garantie pour le financement des petites entreprises et le refinancement des organisations locales de microcrédit et d'épargne et de crédit.

Les organisations de microfinance peuvent en leur sein constituer pour leurs clients ou membres des fonds mutuels de garantie en incluant ce partage du risque dans le taux d'intérêt. Dans un grand nombre de cas, le versement de cette participation au fonds de garantie s'apparente, comme les épargnes bloquées, à une technique permettant d'inscrire le taux d'intérêt pratiqué dans les limites légales du taux d'usure.

10 Voir Jean-Michel Servet, Banquiers aux pieds nus, op. cit., pp. 83 et suiv.

11 Kurt Moors et Geert Peetermans, Fonds de garantie: facteur de succès du microfinancement, Louvain, Cera Foundation, 2002, p. 19. 
Il y a dans une relation de garantie au moins trois parties prenantes:

$\checkmark$ le garant;

$\checkmark$ l'établissement faisant crédit et bénéficiant de la garantie;

๑ l'emprunteur.

A ces trois partenaires peut s'ajouter un quatrième, si une institution de microfinance joue un rôle médiateur permettant de réduire les coûts de transaction tant pour la banque que pour le fonds de garantie lui-même. Il est possible aussi que le fonds de garantie bénéficie de contre-garanties lui permettant d'accroître le capital disponible avec lequel il travaille ${ }^{12}$.

Les organisations de microfinance tout comme les micro-entreprises connaissent une grande difficulté à obtenir des prêts des institutions financières locales, en particulier des banques commerciales, parce le risque de ces crédits paraît trop élevé à ces dernières. La confiance dans les micro-entreprises est limitée parce que le degré d'informalité de leur gestion est élevé, parce qu'elles sont dans l'incapacité de faire la preuve d'un historique de crédit et que, à défaut de protection sociale institutionnalisée, nombre d'entreprises jouent communément un rôle de protection et de solidarité vis-à-vis des membres de la famille de l'entrepreneur et de ses proches. Elles ne présentent pas des comptabilités normées et elles offrent très rarement des biens pouvant être hypothéqués ou gagés ${ }^{13}$. Le niveau comparativement élevé des taux d'intérêt pratiqués dans certaines relations financières dites informelles, en particulier quand prime pour les emprunteurs le besoin immédiat et à court terme de liquidités sur toute autre considération, apparait pour le secteur bancaire plus comme la preuve d'un niveau de risque extrêmement fort que comme un rendement potentiellement très élevé des placements.

Les organismes de microfinance qui interviennent dans ce secteur des microentreprises, jugé risqué par les banques, subissent indirectement ces préjugés défavorables en matière d'évaluation des risques. Cela explique pourquoi les ressources des institutions microfinancières pratiquant ce type de prêt ont plus

12 Ainsi, le capital de travail du FIG (4,5 millions de francs) est constitué pour les deux tiers de son capital social et pour un tiers de contre-garanties. L'objectif du FIG est d'atteindre d'ici 2009 un capital de travail de 10 millions de francs, ce qui pourrait lui permettre de couvrir par les intérêts perçus ses charges de fonctionnement en les répartissant sur un volume d'opérations plus grand.

13 Il nous paraît toutefois erroné, sur la base de tels exemples, de prétendre que le crédit est essentiellement fondé par la mise en gage de la propriété. C'est le cas d'Otto Steiger, «Property Economics versus New Institutional Economics», Journal of Economic Issues, vol. 40, ${ }^{\circ}$ 1, March 2006, pp. 183207, qui dans cet article manifeste une connaissance superficielle de la microfinance en croyant pouvoir appliquer à un thème à la mode, le microcrédit, sa distinction entre propriété et possession. Cette approche néo-institutionnaliste, qui dans certains contextes est pertinente mais qui ne peut pas être ainsi généralisée à chaque usage de l'argent, apparaît comme une conception antékeynésienne et quasi lockienne des rapports économiques. Ce qui, au cœur de la dynamique capitaliste du développement, fonde le crédit est la capacité à évaluer les revenus à venir. C'est la condition essentielle de l'évaluation du risque d'un prêt par un banquier. Cautions et garanties par des titres de propriété viennent couvrir le risque entrepreneurial préalablement estimé, tout comme l'achat d'un bien immobilier ne peut se faire au vu de la seule valeur de l'actif acquis ou des flux de revenus qu'il engendre. Les agents de crédit ont par exemple une propension très forte à prêter aux familles dont certains membres ont migré et envoient régulièrement des fonds... 
souvent pour origine les fonds de donateurs publics et privés ${ }^{14}$ que des placements ou les marchés des capitaux, quoique la microfinance dite commerciale connaisse un essor remarquable qui ne pourra se poursuivre qu'en investissant des couches de populations situées largement au-dessus de la ligne de pauvreté tout en étant en situation d'exclusion bancaire. Le fait qu'une infime minorité des organisations de microfinance fassent preuve d'une rentabilité avérée ne peut que renforcer cette situation ${ }^{15}$. Un fonds de garantie ne s'adresse pas seulement à ces quelques organisations de microfinance rentables. Peu importe que les comptes soient équilibrés grâce à une intervention dans des secteurs de clientèle permettant de couvrir intégralement les coûts de transaction (par exemple du fait de leur niveau de revenu ou de la très forte densité du peuplement) ou que l'équilibre financier soit assuré par des subventions accordées en raison des effets jugés positifs des actions entreprises par les coopérations multilatérales et bilatérales, les pouvoirs publics locaux ou des donateurs. Ce qui compte pour un fonds de garantie, c'est que l'organisation de microfinance qui souscrit un prêt auprès d'une banque soit en capacité de le rembourser, ou que cette organisation présente ou gère des dossiers d'emprunteurs qui seront capables de rembourser intérêt et capital aux échéances prévues.

\section{Différents modèles de garantie}

Il est possible de distinguer trois modèles de garantie selon les liens établis entre les partenaires de la relation financière dans le processus de couverture du risque du prêt et l'octroi de celui-ci, à savoir: le fonds de garantie, l'établissement prêteur, les clients et dans certains cas une institution médiatrice du prêt et de la garantie.

\section{Le modèle de garantie dit «individuel»}

\section{Schéma 1: Le modèle individuel}

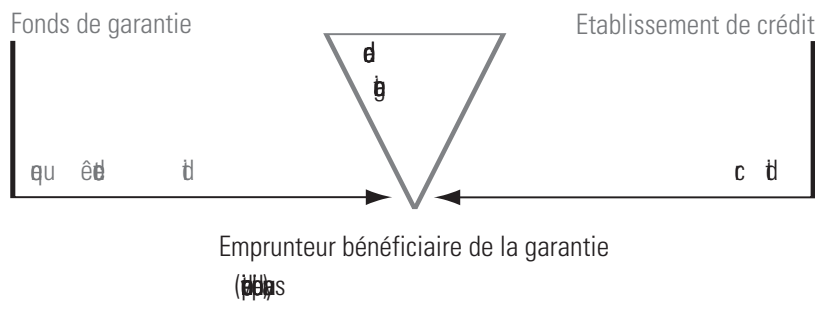

14 Daniel Fino, Le rôle des subventions dans la microfinance, document de travail, projet du Réseau universitaire international de Genève (RUIG) «Efficience, performance financière et rôle des politiques publiques: une enquête sur les conditions de l'autosuffisance financière des institutions de microfinance», Genève, IUED, août 2005, 11 p.

15 Les campagnes des Sommets du microcrédit et le Groupe consultatif d'assistance aux plus pauvres (CGAP) ont tendance à se focaliser sur certains types d'institutions de microfinance nées de transformation d'organisations non gouvernementales ou d'initiatives de banques commerciales. Il est possible de se demander si le paysage de la microfinance n'est pas en fait plus complexe, en y intégrant des structures coopératives locales et de très petites banques privées qui fondent leur solidité non sur la collecte des soutiens internationaux mais sur des relations fortes avec une clientèle épargnante. Cela semble le cas notamment en Asie du Sud et de l'Est. 
Dans le cas d'un modèle de garantie dit «individuel» (voir schéma 1), il existe une relation directe entre le garant (autrement dit le fonds de garantie), le bénéficiaire de la garantie et la banque. Le fonds de garantie accorde une garantie de prêt à un emprunteur potentiel (paysan, petit entrepreneur) en fonction de ses propres critères et de sa connaissance du passé financier ou des caractéristiques de l'emprunteur. Muni d'une lettre de garantie (couvrant en général une fraction seulement du montant du prêt à la suite d'un accord entre le fonds de garantie et l'établissement de crédit), l'emprunteur potentiel s'adresse à un établissement de crédit qui évalue lui aussi cette demande de prêt en fonction de ses propres critères.

En général, le bénéficiaire de la garantie verse une commission au fonds de garantie; le montant de celle-ci dépend du montant du prêt et de la garantie. L'établissement financier consentant le prêt peut dans certains cas percevoir cette commission et la reverser au fonds de garantie.

Entrent dans cette catégorie de garantie individuelle les fonds de garantie organisés en interne par les organisations de microfinance qui agissent comme acteur financier direct.

\section{Le modèle de garantie dit de portefeuille}

\section{Schéma 2: Le modèle de portefeuille}

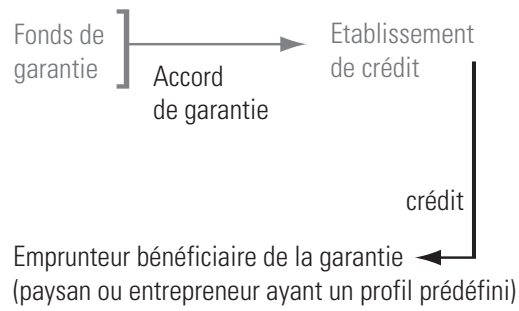

Dans le modèle de garantie dit de portefeuille (voir schéma 2), le fonds de garantie convient avec la banque de se porter garant pour un montant global donné et pour un type déterminé d'emprunteurs potentiels. Ceux-ci doivent répondre à des critères prédéterminés. Il s'agit par exemple du plafond individuel de crédit, des caractéristiques des emprunteurs, de la destination du prêt, etc. La cible peut par exemple être constitué par des micro-entrepreneurs de telle ou telle zone géographique et parfois pour telle ou telle gamme d'activités. Dans ce cas, la relation entre le fonds de garantie et les bénéficiaires de la garantie est généralement indirecte car elle est médiatisée par l'institution faisant le prêt. Le fonds de garantie couvre pour un certain pourcentage du prêt tous les prêts qui lui sont présentés par l'établissement financier pour autant qu'ils répondent aux critères que recherche le fonds et pour un volume global qui, lui aussi, a été fixé par accord avec l'établissement faisant le prêt.

Entre dans cette catégorie l'exemple au Niger du dépôt bloqué et rémunéré dans une banque de la place fait par l'USAID en 1995 au profit de la Coopérative de services d'intermédiation en crédit rural KOKARI pour ouvrir des prêts aux organisations paysannes ${ }^{16}$. Dans les termes du contrat, il était prévu que la

16 Geneviève Nguyen, «Fonds de garantie: intérêts et limites», BIM, n 65, 11 avril 2000, p. 3. 
banque pouvait faire jouer la garantie si les crédits n'étaient pas remboursés trois mois après l'échéance (à hauteur de $90 \%$ des impayés). Dans les faits, la banque n'avait de fait pas prêté plus et cela avait tendu plus à une déresponsabilisation de l'organisation de microfinance et de la banque vis-à-vis des recouvrements qu'à une dynamique de crédit.

\section{Le modèle de garantie par la médiation d'une organisation de microfinance}

\section{Schéma 3: Le modèle de l'intermédiaire}

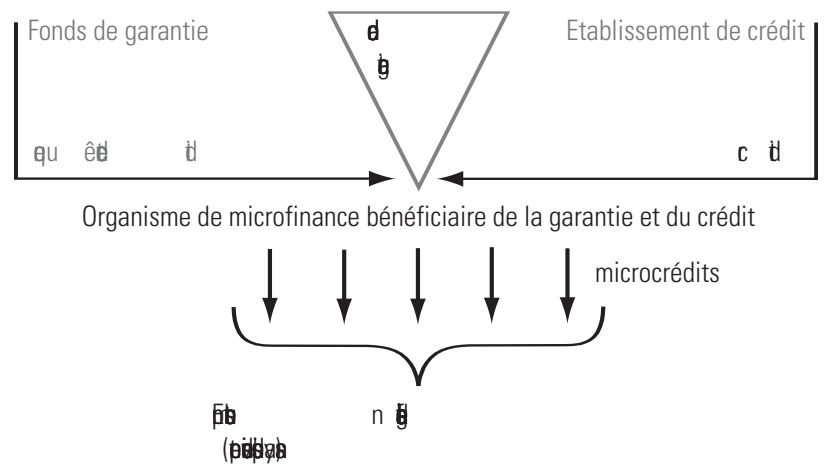

Le troisième type de garantie (voir schéma 3) est celui où une organisation de microfinance obtient la garantie du fonds de garantie et emprunte auprès d'une banque pour reprêter sous forme de microprêts à des micro-entrepreneurs (boutiquiers, vendeurs de rue, artisans, transporteurs, etc.) ou à des paysans. L'organisation de microfinance développe ses propres critères et méthodologies dans la diffusion des prêts.

La Société financière internationale (SFI) par exemple a garanti des lignes de crédit afin de faciliter l'accès au crédit bancaire local par les organisations de microfinance, telle la garantie de $40 \%$ des prêts bancaires par la SFI à la Banque nationale de développement agricole (BNDA) dans le cas de Kafo Jiginew au Mali en 1999; un accord analogue a été passé avec Nyesigiso et avec les caisses villageoises d'épargne et de crédit autogérées (CVECA) du pays dogon. Toutefois, les conditions restrictives pour l'accès à ces garanties au sein des banques locales font que peu d'organisations de microfinance présentent les performances financières exigées pour y avoir accès.

Certains réseaux internationaux de microfinance disposent de leurs propres fonds de garantie, par exemple ACCION, Finca ou Women's World Banking.

Les trois modèles répondent de manière particulière au manque de garantie des prêts par caution, nantissement ou hypothèque et facilitent l'obtention d'un crédit auprès de la banque. Toutefois, le troisième modèle possède une supériorité relative comparativement aux deux précédents. Alors que dans le modèle dit individuel et dans le modèle dit de portefeuille l'établissement prêteur subit le coût de transaction élevé d'un microcrédit, dans le modèle de garantie par l'intermédiaire d'une organisation de microfinance, le coût de transaction élevé (par 
euro prêté) pour des prêts de très faible montant est subi par l'organisation de microfinance et non par le fonds de garantie ou par la banque.

Une banque qui n'a pas une bonne connaissance des milieux micro-entrepreneuriaux peut ainsi pénétrer indirectement ce marché par la médiation de l'organisation de microfinance. On se situe dans un modèle d'adossement financier ${ }^{17}$.

Pour que le système de garantie fonctionne bien, il faut que la procédure de demande de garantie ne soit pas trop lourde et que la réponse à une demande de crédit soit donnée de façon assez rapide. A l'inverse, il faut que, en cas de défaillance d'un emprunteur, l'institution bénéficiaire de la garantie obtienne tout aussi rapidement ce qui lui est dû. Cela suppose l'établissement de bonnes relations entre les partenaires de l'opération.

\section{L'exemple du Fonds international de garantie de Genève}

Le Fonds international de garantie (FIG) est une expérience originale en matière de garantie et illustre la capacité de rompre avec la logique de l'apport extérieur de fonds ainsi que celle de valoriser et de dynamiser les ressources locales en s'appuyant sur des partenariats à différents niveaux. Cette société coopérative à but non lucratif a été fondée à Genève en décembre 1996 par plusieurs partenaires du Nord et du Sud, notamment une fondation suisse, «Recherches et applications de financements alternatifs au développement» (RAFAD) ${ }^{18}$, à laquelle se sont progressivement associées des municipalités, la coopération suisse, la fondation Soros, des personnes privées, etc. ${ }^{19}$ La ville de Genève présente une des densités les plus élevées d'organisations non gouvernementales de solidarité internationale ${ }^{20}$ au monde. Cela tient sans doute aux spécificités de la coopération et de la solidarité suisses et au poids local des institutions des Nations unies présentes dans la ville ${ }^{21}$. Sur une plus longue période et de façon générale dans le champ de l'économie sociale et solidaire, on observe que des initiatives alternatives helvétiques ont su se pérenniser en fonctionnant sur des principes d'adaptation et de dialogue et non de rupture avec leur environnement

17 Cette expression traduit ici l'anglais bank linkage.

18 La fondation RAFAD a été créée en 1985 par des praticiens du développement avec pour mission de contribuer au développement des économies locales des pays du Sud en évitant les dépendances de l'aide traditionnelle au développement. Le FIG a son siège dans les locaux de la fondation RAFAD. Celle-ci participe à la Fédération genevoise de coopération (FGC), qui a été créée en 1966 et qui regroupe une cinquantaine d'associations ayant un siège dans le canton de Genève et qui agissent dans le domaine de la coopération internationale au développement et dans l'information du public sur les rapports Nord-Sud et le développement. Ce n'est pas un organe de financement direct mais un espace de dialogue et d'échanges. La FGC est un interlocuteur privilégié du canton de Genève, de ses municipalités et du Département fédéral des affaires étrangères (DFAE) à Berne. Une vingtaine de communes du canton financent des projets à travers elle.

19 Les associés du FIG sont 38 personnes privées, toutes originaires du Nord, 33 institutions du Sud et 9 institutions du Nord. Je remercie ici particulièrement Daniel Fino et Fernand Vincent, administrateurs du FIG, pour les informations communiquées. Voir Philippe Berberat, Daniel Fino et François Mercier, «Instrument de la garantie internationale au service des plus pauvres», Annuaire SuisseTiers Monde 2001, Genève, IUED, 2002, pp. 69-81, et les rapports annuels d'activité du FIG.

20 Voir la liste sur <http://www.mandint.org >. Sur l'économie sociale et solidaire à Genève, voir le site de l'Association pour la promotion de l'économie sociale et solidaire (APRÈS), <http://www.apresge.ch>.

${ }^{21}$ Par exemple, 170 ONG ayant un statut consultatif auprès de l'ONU y sont établies. 
social et culturel. La ville compte aussi une des plus fortes concentrations de gestion de fortunes privées au monde. D'où un certain pragmatisme et des innovations financières ${ }^{22}$.

Le principal objectif du FIG est de permettre d'aider des institutions de microfinance et des coopératives agricoles dans les pays en développement à obtenir des prêts en monnaie locale de la part de banques commerciales locales, et donc de favoriser l'accès au crédit de micro-entrepreneurs individuels ou coopérateurs des pays en développement. Il aurait au cours de ses dix années d'activités contribué à créer plus de 250000 emplois. Depuis 1996, le FIG a collaboré étroitement avec 53 institutions de microfinance et coopératives agricoles situées dans 17 pays; actuellement il est actif dans cinq pays d'Afrique et six d'Amérique latine ${ }^{23}$. Au lieu d'intervenir directement sous forme de transferts unilatéraux de fonds, ensuite prêtés selon la technique du microcrédit, le FIG facilite l'accès aux institutions financières locales par le biais d'une garantie de leurs prêts. Il ne s'agit donc pas de transférer des ressources de pays développés à des pays supposés moins développés et manquant cruellement de ressources financières, mais d'encourager, grâce à cette garantie extérieure permettant la diminution du risque pris par la banque locale, une intermédiation financière courte et une mobilisation de ressources financières inactives au Sud. Une des principales raisons de la mise en place de ce dispositif est le constat que nous avons fait au début de cet article qu'un certain nombre d'établissements financiers ne prêtent pas, non par manque de ressources pour s'engager mais parce qu'ils ne veulent pas prendre le risque de le faire, compte tenu de leur incapacité d'évaluer les entreprises et les institutions qui les sollicitent (défaillance des comptabilités, manque d'historique des relations bancaires, incapacité de faire intervenir les tribunaux, absence de marché pour vendre les biens pris en gage, etc.). Il est à souligner qu'il s'agit avec le FIG d'un risque partagé entre les différents partenaires de cette relation, et entre institutions du Nord et du Sud.

Pour l'illustrer, prenons un exemple. Une association locale d'épargne et de prêt veut accroître ses fonds disponibles destinés à être prêtés à ses membres; une banque commerciale locale serait disposée à lui ouvrir un crédit équivalent à 100000 euros, sous réserve que l'association présente une garantie pour la moitié de cette somme. L'association peut bénéficier d'une garantie du FIG pour autant qu'elle en soit ou en devienne membre, qu'elle ait acquis au moins le dixième du montant de la garantie en parts sociales du fonds et que, après approbation du dossier par le comité de garantie du fonds, elle lui verse chaque année une commission s'élevant entre $3 \%$ et $5 \%$ du montant de la garantie (frais bancaires inclus). Les parts versés au FIG tant par les partenaires du Nord que du Sud ne donnent pas lieu à rémunération. Ajoutons que la banque locale ne reçoit pas directement du FIG la garantie accordée à l'association qui emprunte auprès d'elle; celle-ci transite par l'Union de Banques Suisses (UBS), une des principales institutions financières helvétiques. Cette notoriété accroît

22 Ce que rappelle hors Genève le cas du WIR, système d'échange local, né dans les années 1930 et encore dynamique septante ans après leur création en Suisse alémanique. Il a ouvert récemment une représentation à Lausanne. Des initiatives similaires en Allemagne, en Autriche ou en France ont très rapidement disparu... pour renaître sous d'autres formes quelques années plus tard (voir J.-M. Servet [dir.], Une économie sans argent, Paris, Seuil, 1999).

23 Ces pays sont le Bénin, le Burkina Faso, le Rwanda, le Sénégal et le Togo d'une part et le Chili, l'Equateur, le Guatemala, le Nicaragua, le Pérou et le Salvador d'autre part. 
considérablement la force de la garantie et ainsi la confiance dans la relation qui s'établit ${ }^{24}$. On doit remarquer aussi qu'il s'agit d'une garantie partagée entre l'institution qui prête localement et le FIG; l'effet multiplicateur est actuellement estimé à cinq, autrement dit la garantie de ce fonds permet d'obtenir un prêt d'un montant cinq fois plus élevé que les sommes immobilisées par la garantie. L'absence de remboursement d'un prêt qui serait consenti par un établissement du Nord évite tant l'alourdissement de la dette du fait de l'appréciation des monnaies fortes que les blocages du fait d'un contrôle des changes imposé par certains pays; ceux-ci rendent impossibles les remboursements d'un crédit direct, par exemple en Equateur et au Rwanda. La dette est souscrite en monnaie locale et la garantie est faite pour partie en francs suisses, en euros ou en dollars par le FIG et en monnaie locale par l'institution qui consent le prêt.

Ajoutons que cette garantie peut jouer en faveur d'une diminution du taux d'intérêt exigé par l'institution locale ouvrant le prêt. Toutefois on remarque que le prêt obtenu grâce à cette garantie peut l'être à un taux plus élevé qu'un crédit consenti par un fonds international de placement à finalité lucrative. Cela provient du fait que, même si le taux d'intérêt du prêt local obtenu grâce à la garantie et celui du prêt d'un crédit international sont de même niveau, au coût du prêt lui-même s'ajoute celui de l'immobilisation de la participation au fonds et de la commission annuelle. Or la somme immobilisée pour participation au fonds l'est pendant toute la durée d'un prêt alors que les intérêts portent sur le seul capital restant dû. C'est souvent parce que l'institution locale de microfinance ne peut pas obtenir un prêt qu'elle s'adresse au fonds de garantie. A cela s'ajoute que la ressource immobilisée dans la participation est souvent constituée à la suite d'un don fait dans ce but à la structure. Celle-ci peut la récupérer en fin de garantie alors que le crédit acquitté est définitivement perdu. On ne peut pas dans ces circonstances considérer cette somme de façon systématique comme une ressource additionnelle coûteuse et comparer à court terme le coût de deux sources différentes de financement qui pourraient potentiellement être obtenues de façon concurrente. Si tel était le cas, on ne comprendrait pas la raison pour laquelle une institution de microfinance préfère un prêt garanti et plus coûteux à un prêt moins cher et qui ne l'est pas.

La garantie prend fin avec le remboursement de la totalité du prêt. On peut remarquer toutefois que la relation ainsi établie est poursuivie avec d'autres crédits et que s'engage une relation durable fondée sur la connaissance par le FIG de ses partenaires. Les placements faits par lui grâce aux sommes reçues sont gérés de façon éthique ou socialement responsable. Les intérêts de ces placements contribuent à couvrir les frais de gestion à Genève et sur le terrain, où le FIG a deux représentants régionaux, l'un au Nicaragua et l'autre au Bénin, eux-mêmes appuyés par dix consultants locaux. Il faudrait que le volume d'activités du FIG soit plus que doublé pour qu'il parvienne à couvrir totalement ses frais de fonctionnement. La pérennité de ce type de fonds dépend du suivi de la discipline des remboursements par chacun des partenaires. Le partage des risques entre les différents niveaux, loin de diluer les responsabilités, devrait éviter qu'un fonds de garantie de ce type ne subisse le même sort que dans le cadre des politiques de crédit public, en particulier celles à destination de l'agriculture dans les années 1960 et 1970.

24 Pour une approche conceptuelle de la confiance et son application au domaine financier, voir: Philippe Bernoux et Jean-Michel Servet (dir.), La construction sociale de la confiance, Paris, Montchrestien; Association d'économie financière (AEF), 1997. 
Schéma 4: Effet multiplicateur de la garantie du FIG

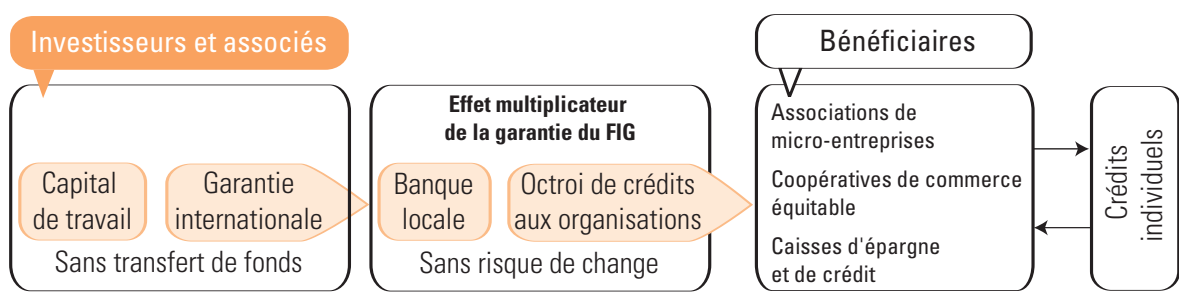

\section{Conclusion: une mobilisation par la garantie de la solidarité entre institutions et avec la clientèle}

On doit souligner que la garantie institue une solidaritée 25 entre les divers partenaires de la relation de garantie. La solidarité la plus évidente se situe dans la répartition et le partage des risques.

Un fonds de garantie s'appuyant sur une organisation de microfinance permet de nouer des liens entre le secteur financier et les micro-emprunteurs. Il s'agit tant d'un processus d'apprentissage de mécanismes financiers par les emprunteurs et d'une capacité de négocier en la matière que d'un apprentissage du respect des engagements financiers pris. A l'inverse, la mise en place de garanties assurant un investissement des banques dans des prêts à destination de microentreprises permet au secteur financier traditionnel d'acquérir une meilleure connaissance des activités du secteur des micro-entreprises.

Pour qu'une garantie fonctionne de façon satisfaisante, il est indispensable que le risque soit bien réparti entre les trois parties concernées: le fonds de garantie, l'établissement faisant le prêt et le bénéficiaire de la garantie. La banque locale doit partager une partie du risque, car elle peut chercher à reporter sur le fonds de garantie toutes les demandes de crédit qui lui paraissent les plus risquées et ne conserver que les dossiers qu'elle juge à risque limité. Si la banque locale ne partage pas une partie du risque, elle peut aussi avoir tendance à adopter une attitude laxiste dans le suivi des remboursements ${ }^{26}$.

Un deuxième élément à souligner est l'effet de levier et l'effet multiplicateur qui naissent de la solidarité induite par la garantie. Le système de la garantie augmente le volume de fonds mis à disposition pour faire l'objet de prêt. Une garantie externe à un pays en développement permet une mobilisation de ressources financières dans ce pays à partir d'une mobilisation de ressources au Nord et qui ne font pas l'objet d'un transfert international de fonds. C'est l'effet de levier. A cela s'ajoute un effet multiplicateur ${ }^{27}$ : les dons affectés à un fonds de garantie permet-

25 Pour une définition de la solidarité à partir de l'approche polanyienne de la réciprocité, nous renvoyons à notre contribution «Le principe de réciprocité chez Karl Polanyi », Revue Tiers Monde, $\mathrm{n}^{\circ} 190$, août 2007, pp. 255-274.

26 Voir ci-dessus l'exemple donné à propos d'une ligne de crédit garantie par l'USAID au Niger.

27 Dans la documentation du FIG est appelé «effet de levier» ce que nous désignons ici par «effet multiplicateur », que nous distinguons de l'effet initial de mobilisation de ressources (qualifié ici d' «effet de levier »). 
tent un montant de prêt plus grand que si ces dons étaient transférés au Sud et y étaient consacrés directement à des prêts ou a fortiori s'ils n'étaient pas remboursés. Dans la mesure où le dépôt du fonds permet à l'établissement financier du Nord, qui reçoit le dépôt de la garantie, de bénéficier de ressources elles-mêmes prêtables, l'effet multiplicateur peut être plus grand encore (l'immobilisation du fonds dépend du degré de risque estimé et couvert). Les dépôts de garantie peuvent par exemple alimenter des placements éthiques et les revenus de ceux-ci couvrir les frais de fonctionnement du fonds. Ainsi, à la solidarité entre les territoires et au partage des risques et des richesses entre groupes sociaux (caractéristiques de la solidarité du fonds de garantie lui-même) peuvent, du fait de ces placements éthiques, s'ajouter d'autres types de solidarité au sein des territoires, entre les générations, entre sexes et avec les générations futures dans la perspective d'un développement socialement durable et physiquement soutenable.

On doit aussi noter que le fonds de garantie permet de limiter les effets de la détérioration des taux de change (en comparaison d'un prêt accordé en devise étrangère). Le prêt étant accordé en monnaie locale (à partir d'une garantie réalisée par un organisme situé à l'extérieur), l'emprunteur doit rembourser à partir de ses propres capacités locales elles aussi et non dans une monnaie forte dont le taux d'appréciation s'ajoute au taux d'intérêt lui-même. Quand un prêt est accordé en monnaie locale à partir d'une ressource exprimée dans une monnaie dite «forte», le taux d'intérêt est nécessairement plus élevé puisqu'il inclut dans le remboursement le risque de change et la détérioration probable de la monnaie nationale. Or, un taux d'intérêt élevé accroît le coût du crédit et par là diminue les capacités de remboursement de l'emprunteur et augmente son risque de défaillance. Que le prêt soit accordé en monnaie locale doit aussi permettre d'accroître progressivement, en en diminuant le risque, sa taille et sa durée. Cela n'est pas une nécessité car il est faux de penser que les micro-entrepreneurs aient en tout lieu et en tout temps besoin d'un volume croissant de prêt. Si la monnaie locale se déprécie par rapport à la devise dans laquelle à l'extérieur a été constitué le fonds de garantie, le volume ultérieur de prêts réalisables en monnaie locale (qui se déprécie) s'accroît. Un fonds international de garantie ne subit pas alors les conséquences directes de l'érosion de monnaies nationales. Toutefois, un fonds de garantie n'est pas à l'abri des effets de dépréciations monétaires des dépôts réalisés en devises sur ses comptes par ses partenaires ${ }^{28}$. Il ne s'agit bien évidemment pas alors d'un déficit d'exploitation. L'appréciation d'une monnaie peut provoquer un effet inverse, ce qui ne traduit pas une meilleure gestion du fonds. Cela illustre le risque lui-même de l'intermédiation des risques.

Si la relation s'inscrit dans le temps, il est possible d'espérer que plus ou moins rapidement une relation durable de confiance s'instaurera entre l'institution de microfinance faisant le prêt et le secteur bancaire local, permettant que, à terme, les opérations de déblocage des prêts soient plus rapides, que la durée des prêts consentis soit allongée, que les exigences en matière de garantie fournie soient moins fortes (afin d'accélérer les demandes d'acceptation et de versement), etc., et même qu'il n'y ait plus besoin de garantie extérieure et que l'établissement bancaire ouvrant le prêt accepte de prendre le risque de celui-ci. On doit noter

28 En 2004, du fait d'une perte de valeur du dollar américain de $30 \%$ en deux ans, le FIG a connu une perte comptable d'un peu plus de 50000 francs suisses. Une situation inverse a été constatée l'année suivante. 
que plus le pourcentage du prêt garanti par le fonds extérieur diminue et plus la banque locale accepte de couvrir un pourcentage élevé, plus l'effet de levier des ressources locales s'accroît et plus l'effet multiplicateur est grand. Si le pourcentage du prêt garanti diminue, le volume de prêt pouvant être garanti s'accroît: c'est l'effet de multiplication. C'est-à-dire que le fonds est à même de garantir deux fois plus, trois fois plus, etc., de prêts. En quelque sorte, le succès d'un fonds de garantie du Nord vis-à-vis du Sud est de faire en sorte que son action soit temporaire et décroissante et que son besoin disparaisse le plus vite possible. Il doit être par nature un instrument de transition et permettre la construction de relations financières nouvelles au $\mathrm{Sud}^{29}$.

Alors que les transferts internationaux de fonds sous forme d'aide (qu'il s'agisse de prêts ou de dons) s'inscrivent fondamentalement dans une logique de protection, les fonds internationaux de garantie peuvent activer avec une intensité variable des mécanismes de réciprocité et de solidarité. Ceux-ci se caractérisent essentiellement par le fait que la relation entre partenaires est établie et pratiquée sur un pied d'égalité et que ces derniers agissent en toute conscience comme étant en interdépendance volontaire.

\section{Bibliographie}

Agbodjan, Djimitri Edoé, La microfinance dans l'après-conflit, mémoire de DEA, Genève, IUED, 2006.

Bastiaenen, Michiel and Peter van Rooij, Guarantee Funds and NGOs: Promise and Pitfalls, Povertyoriented Banking Working Paper, $n^{\circ}$ 18, Geneva, International Labour Office (ILO), 1997, 19 p.

Berberat, Philippe, Daniel Fino et François Mercier, «Instrument de la garantie internationale au service des plus pauvres », Annuaire Suisse-Tiers Monde 2001, Genève, IUED, 2002, pp. 69-81.

Bernoux, Philippe et Jean-Michel Servet (dir.), La construction sociale de la confiance, Paris, Montchrestien; Association d'économie financière (AEF), 1997, 385 p.

Fino, Daniel, Le rôle des subventions dans la microfinance, document de travail, projet du Réseau universitaire international de Genève (RUIG) «Efficience, performance financière et rôle des politiques publiques: une enquête sur les conditions de l'autosuffisance financière des institutions de microfinance», Genève, IUED, août 2005, 11 p.

King, Bradley, MFI Guarantees: Economic and Marketing Research Findings, Working Paper, ES Entreprising Solutions Global Consulting, 31 March 2005, 19 p.

Lopez, Cesar and Jorge de Angulo, «Bridging the Finance Gap: ACCION's Experience with Guarantee Funds for Microfinance Institutions », InSight, no 15, Boston, ACCION, September 2005, 18 p.

Moors, Kurt et Geert Peetermans, Fonds de garantie: facteur de succès du microfinancement, Louvain, Cera Foundation, 2002, 56 p.

Nguyen, Geneviève, «Fonds de garantie: intérêts et limites », BIM, nº 65, 11 avril 2000, 8 p.

Servet, Jean-Michel, Banquiers aux pieds nus. La microfinance, Paris, Odile Jacob, 2006.

Sgard, Jérôme, Rapport sur le risque-pays du Maroc, Document de travail, nº 18, Paris, Agence française de développement, juin 2006, 33 p.

Steiger, Otto, «Property Economics versus New Institutional Economics », Journal of Economic Issues, vol. 40, nº 1, March 2006, pp. 183-207.

Techniques financières et développement (revue d'Epargne sans frontière, Paris), $\mathrm{n}^{\mathrm{o}} 36$, septembreoctobre 1994.

29 On peut remarquer que le Fonds international de garantie de Genève a été à l'origine en 2004 du Fonds international de garantie Amérique latine (FIGAL) au Nicaragua. La création du FIGAL correspond à un processus de décentralisation qui a été réalisé en partenariat avec l'American Foundation et doit étendre ses activités dans d'autres pays d'Amérique centrale. Un projet similaire est en cours d'élaboration au Bénin. 